\title{
Cristalização de superfície em vidro do sistema $\mathrm{Li}_{2} \mathrm{O}-\mathrm{ZrO}_{2}-\mathrm{SiO}_{2}-\mathrm{Al}_{2} \mathrm{O}_{3}$
}

\author{
Surface crystallization in a \\ $\mathrm{Li}_{2} \mathrm{O}-\mathrm{ZrO}_{2}-\mathrm{SiO}_{2}-\mathrm{Al}_{2} \mathrm{O}_{3}$ glass
}

Antonio Pedro Novaes de Oliveira ${ }^{1}$, Alexandre Henrique Bortolotto Teixeira ${ }^{1}$, Hugo Henrique Venturelli ${ }^{1}$, Oscar Rubem Klegues Montedo ${ }^{2}$

\footnotetext{
${ }^{1}$ Laboratório de Materiais Vitrocerâmicos - VITROCER/PGMAT/UFSC 88040-900, Florianópolis, SC

e-mail: bortolotto_teixeira@yahoo.com.br; venturellihugo@gmail.com; antonio.pedro@ufsc.br

${ }^{2}$ Grupo de Pesquisa em Cerâmica Técnica - CERTEC/PPGCEM/UNESC 88806-000, Criciúma, SC

e-mail: oscar.rkm@gmail.com
}

\begin{abstract}
RESUMO
A cinética de crescimento da camada superficial cristalizada em um vidro de composição do sistema LZSA, $11,7 \mathrm{Li}_{2} \mathrm{O} \cdot 12,6 \mathrm{ZrO}_{2} \cdot 68,6 \mathrm{SiO}_{2} \cdot 7,1 \mathrm{Al}_{2} \mathrm{O}_{3}(\%$ massa $)$, foi estudada. Para a produção do vidro, utilizou-se matérias-primas comerciais $\left(\mathrm{Li}_{2} \mathrm{CO}_{3}, \mathrm{ZrSiO}_{4}, \mathrm{SiO}_{2}, \mathrm{Al}_{2} \mathrm{O}_{3}\right)$, as quais foram homogeneizadas e fundidas a $1550{ }^{\circ} \mathrm{C}$ por 120 min e então vazadas em molde metálico. Amostras do vidro obtido foram seccionadas e submetidas a tratamentos térmicos em diferentes temperaturas $\left(825-925^{\circ} \mathrm{C}\right)$ e tempos $(30-150 \mathrm{~min})$ para formação e crescimento da camada cristalina. Seções transversais das amostras tratadas termicamente foram lixadas e polidas, tal que imagens das camadas cristalizadas pudessem ser visualizadas e medidas por microscopia. Os resultados mostraram que é possível obter vidros do sistema LZSA com camadas cristalizadas contendo principalmente espodumênio- $\beta$ e silicatos de zircônio e lítio, de espessuras entre 13 e $665 \mu \mathrm{m}$, as quais crescem com velocidades entre 0,4 e $4,8 \mu \mathrm{m} / \mathrm{min}$ no intervalo de temperatura estudado.
\end{abstract}

Palavras-chave: Vidros, cinética de cristalização, cristalização de superfície.

\section{ABSTRACT}

Growth kinetics of crystallized surface layer in a LZSA glass composition, $11.7 \mathrm{Li}_{2} \mathrm{O} \cdot 12.6 \mathrm{ZrO}_{2} \cdot 68.6 \mathrm{SiO}_{2} \cdot 7.1 \mathrm{Al}_{2} \mathrm{O}_{3}(\mathrm{wt} \%)$, was studied. For the production of the LZSA glass, it was used commercial raw materials $\left(\mathrm{Li}_{2} \mathrm{CO}_{3}, \mathrm{ZrSiO}_{4}, \mathrm{SiO}_{2}, \mathrm{Al}_{2} \mathrm{O}_{3}\right)$ which were mixed and melted at $1550{ }^{\circ} \mathrm{C}$ for $120 \mathrm{~min}$ and then poured into a metallic mold. Samples of the obtained glass were cut and subjected to heattreatments at different temperatures $\left(825-925^{\circ} \mathrm{C}\right)$ and times $(30-150 \mathrm{~min})$ for formation and growth of crystalline layer. Cross-sections of the heat-treated samples were ground and polished such that images of the formed crystallized layers could be visualized and measured by microscopy. Results showed that it is possible to obtain LZSA glasses with crystallized layers formed by $\beta$-spodumene, zircon and lithium silicate, which present thicknesses between 13 and $665 \mu \mathrm{m}$ and grow at rates varying from 0.4 to $4.8 \mu \mathrm{m} / \mathrm{min}$ in the studied temperature range.

Keywords: Glasses, crystallization kinetics, surface crystallization.

\section{INTRODUÇÃO}

Vidros são, em teoria, mecanicamente muito resistentes. De fato, a resistência mecânica teórica dos vidros, excluindo defeitos e considerando assim apenas a influência das ligações químicas, é de aproximadamente $7000 \mathrm{MPa}$ [1]. Todavia, vidros comerciais têm resistência mecânica usualmente entre 35 e $70 \mathrm{MPa}$ [2, 3]. Tal fato está relacionado a presença de defeitos, principalmente defeitos superficiais. $\mathrm{O}$ ambiente e a temperatura também influenciam nessa propriedade [2,4]. É possível melhorar a resistência mecânica de vidros de diversas maneiras, como por exemplo:

- eliminando as falhas superficiais por meio de polimento a quente, resultando em um aumento temporário da resistência mecânica a partir do momento em que o material é colocado em serviço;

- depositando um revestimento protetor; ou 
- introduzindo tensões superficiais compressivas na superfície [5].

A geração de tensões de compressão na superfície dos componentes vítreos é a maneira mais eficiente de se obter aumento considerável em sua resistência mecânica [1]. Neste caso, a profundidade da camada compressiva deve ser maior que as falhas superficiais presentes, isto é, maior ou igual a $50 \mu \mathrm{m}[1,6,7]$. Como a maioria das falhas nos vidros alcançam dimensões entre 1 e $10 \mu \mathrm{m}$, Varshneya [8] sugere que a profundidade ou espessura da camada compressiva deva ser de aproximadamente $30 \mu \mathrm{m}$ para promover efetiva proteção contra fratura $[9,10]$. Atualmente, os principais processos conhecidos de geração de tensões compressivas na superfície de componentes de vidro são a têmpera térmica, a têmpera química ou troca iônica, a esmaltação, e a cristalização de superfície [8]. Dentre estes métodos, a cristalização de superfície é a menos estudada do ponto de vista cinético, dada a complexidade do seu entendimento.

A medida das taxas de crescimento de camadas cristalinas a partir da superfície livre de vidros é uma técnica experimental bem consolidada [1], o que permite a obtenção de resultados confiáveis e reprodutíveis. Em uma etapa final, permite também a definição de ciclos térmicos que originarão produtos de vidro com camadas cristalizadas de espessuras controladas e, assim, com propriedades adequadas para uma aplicação específica. Na literatura internacional existem muitos estudos completos, referentes a algumas composições de vidros, sobre a cinética de cristalização de superfície e crescimento de camadas cristalinas.

Entretanto, nenhum estudo foi realizado em composições do sistema $\mathrm{Li}_{2} \mathrm{O}-\mathrm{ZrO}_{2}-\mathrm{SiO}_{2}-\mathrm{Al}_{2} \mathrm{O}_{3}$ ( $\mathrm{LZSA}$ ), em particular com relação a determinação da taxa de crescimento da camada cristalina na superfície do vidro produzido.

Neste contexto, este trabalho teve como objetivo o estudo da cinética de crescimento da camada cristalizada em um vidro do sistema LZSA, já que a formação e o controle da espessura desta camada pode resultar em componentes de vidro com maior resistência mecânica pela geração de tensões de compressão na superfície do vidro tratado termicamente.

\section{MATERIAIS E MÉTODOS}

A Figura 1 mostra um fluxograma referente às principais etapas experimentais definidas para a realização deste trabalho, as quais são descritas a seguir.

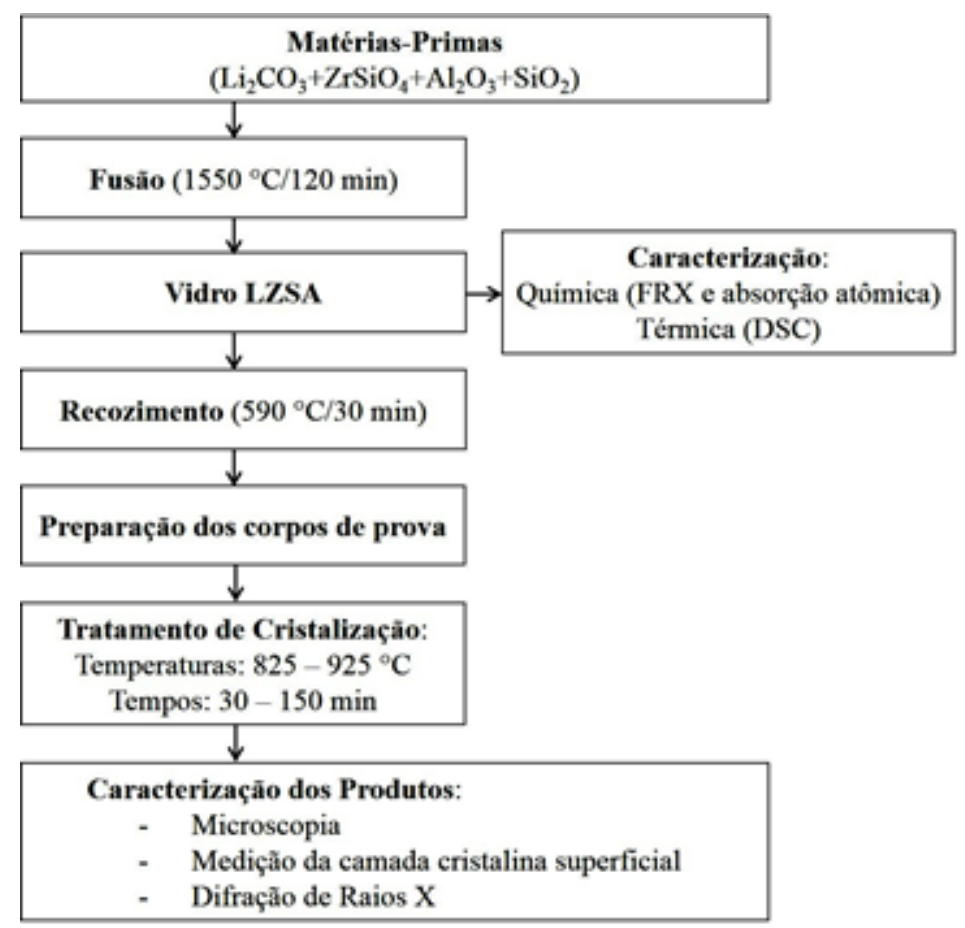

Figura 1: Fluxograma mostrando as principais etapas experimentais deste trabalho.

Para a realização deste trabalho, bateladas de um vidro de composição/formulação $11,7 \mathrm{Li}_{2} \mathrm{O} .12,6 \mathrm{ZrO}_{2} \cdot 68,6 \mathrm{SiO}_{2} .7,1 \mathrm{Al}_{2} \mathrm{O}_{3}(\%$ massa $)$ foram preparadas a partir de matérias-primas de alta pureza 
(> 99\%) disponíveis comercialmente, isto é, carbonato de lítio $\left(\mathrm{Li}_{2} \mathrm{CO}_{3}\right)$, silicato de zircônio $\left(\mathrm{ZrSiO}_{4}\right)$, quartzo $\left(\mathrm{SiO}_{2}\right)$ e alumina $\left(\mathrm{Al}_{2} \mathrm{O}_{3}\right)$. Cada batelada, para produzir $100 \mathrm{~g}$ de vidro, foi misturada a seco em moinho horizontal (Servitech, CT-248) por $1 \mathrm{~h}$ e então colocada em um cadinho de platina e fundida a $1550 \pm 5{ }^{\circ} \mathrm{C}$, em forno elétrico tipo elevador (Jung - Modelo LF0917) por $2 \mathrm{~h}$. O fundido foi, em uma etapa posterior, vazado em um molde de aço (pré-aquecido a $400{ }^{\circ} \mathrm{C}$ ) de formato retangular com dimensões internas nominais de $100 \mathrm{~mm}$ de comprimento $\mathrm{x} 20 \mathrm{~mm}$ de largura $\times 10 \mathrm{~mm}$ de profundidade.

Subsequentemente, uma parte do vidro obtido foi moída em moinho planetário (Retsch, PM-100) utilizando-se jarro e esferas de ágata. O pó obtido foi destinado à análise química, realizada por meio da técnica de fluorescência de raios X, em um equipamento Philips modelo PW 2400, com tubo de $3 \mathrm{~kW}$ e alvo de ródio. A fração de lítio foi determinada por absorção atômica em um equipamento Unican modelo 969 . Outra parte do vidro foi moída em um moinho de bolas de alumina (Servitech, CT-242), atingindo tamanho de partícula inferior a $20 \mu \mathrm{m}$. O pó resultante destinou-se à análise de calorimetria exploratória diferencial (DSC), realizada em um equipamento TA Instruments (SDTQ 600), em atmosfera oxidante, a uma taxa de aquecimento de $10^{\circ} \mathrm{C} / \mathrm{mim}$ utilizando amostra em pó de $30 \mathrm{mg}$ (colocada em cadinho de platina) com um cadinho de platina vazio como material de referência.

Para promover o alívio de tensões de origem térmica no vidro produzido, as barras de vidro moldadas foram recozidas em um forno tipo mufla (EDG 3000) a $590{ }^{\circ} \mathrm{C}$ por $30 \mathrm{~min}$. A partir das barras de vidro produzidas, 35 corpos de prova com dimensões nominais de $20 \mathrm{~mm}$ x $20 \mathrm{~mm} \times 10 \mathrm{~mm}$ foram produzidos mediante corte com o auxílio de uma cortadeira ceramográfica com disco diamantado (Isomet 1000). Em uma etapa posterior, os corpos de prova foram aquecidos a $10^{\circ} \mathrm{C} / \mathrm{min}$ em um forno tipo mufla (EDG 3000) com patamar diferentes temperaturas $\left(825,850,875,900\right.$ e $\left.925^{\circ} \mathrm{C}\right)$ por diferentes tempos de permanência na temperatura $(30,60,90,120$ e $150 \mathrm{~min})$.

Posteriormente, os corpos de prova tratados termicamente foram seccionados (Isomet 1000), embutidos em resina acrílica e, então, lixados e polidos com pasta de alumina $(1 \mu \mathrm{m})$, tal que as camadas cristalizadas puderam ser visualizadas em microscópio ótico (MO, Leica, DM400M com câmera Leica DC 300 e interface com computador) e eletrônico de varredura (MEV, JEOL, JSM-6390LV) e medidas com o auxílio do software ImageJ. Analisando-se os resultados obtidos em função do tempo de tratamento térmico, verificouse um comportamento linear. Assim, determinou-se as linhas de tendência por meio de regressão linear, o que possibilitou a determinação das taxas de crescimento das camadas cristalizadas, as quais representam os coeficientes angulares das equações determinadas.

Por fim, foram identificadas as fases cristalinas formadas nas camadas cristalizadas. Neste caso, corpos de prova (com espessuras nominais de $5 \mathrm{~mm}$ ) tratados termicamente a 825 e $900{ }^{\circ} \mathrm{C}$ por 120 min foram submetidos a análises de difração de raios $\mathrm{X}$ em um difratômetro Philips - X'Pert, com radiação de $\mathrm{CuK} \alpha$, no intervalo de medição (20) entre 3 e $118^{\circ}$, com passo de $0,02^{\circ}$. Para identificação das fases cristalinas formadas, utilizou-se o banco de dados JCPDS.

\section{RESULTADOS E DISCUSSÃO}

A Tabela 1 mostra o resultado da análise química do vidro do sistema LZSA produzido neste trabalho.

Tabela 1: Composição química teórica e analisada do vidro LZSA.

\begin{tabular}{ccc}
\hline \multirow{2}{*}{ Óxidos constituintes } & \multicolumn{2}{c}{ Composição do vidro (\% massa) } \\
\cline { 2 - 3 } & Teórica & Analisada \\
\hline $\mathrm{Al}_{2} \mathrm{O}_{3}$ & 7,10 & 7,44 \\
$\mathrm{CaO}$ & --- & 0,08 \\
$\mathrm{Fe}_{2} \mathrm{O}_{3}$ & --- & 0,07 \\
$\mathrm{Li}_{2} \mathrm{O}$ & 11,70 & 9,90 \\
$\mathrm{Na}_{2} \mathrm{O}$ & -- & 0,12 \\
$\mathrm{SiO}_{2}$ & 68,60 & 68,60 \\
$\mathrm{SrO}_{2}$ & --- & 0,10 \\
$\mathrm{ZrO}_{2}$ & 12,60 & 13,63 \\
$\mathrm{Total}$ & 100,0 & 100,00 \\
\hline
\end{tabular}


Como pode ser verificado pela análise da Tabela 1, há uma pequena diferença entre os valores da composição química teórica e aquela determinada para o vidro produzido, devido à presença de impurezas. Também é possível observar uma pequena diminuição no percentual referente ao óxido de lítio, provavelmente em decorrência da evaporação deste no momento da sua fusão, como comumente reportado na literatura. A Figura 2 mostra a curva de calorimetria exploratória diferencial do pó de vidro do sistema LZSA.

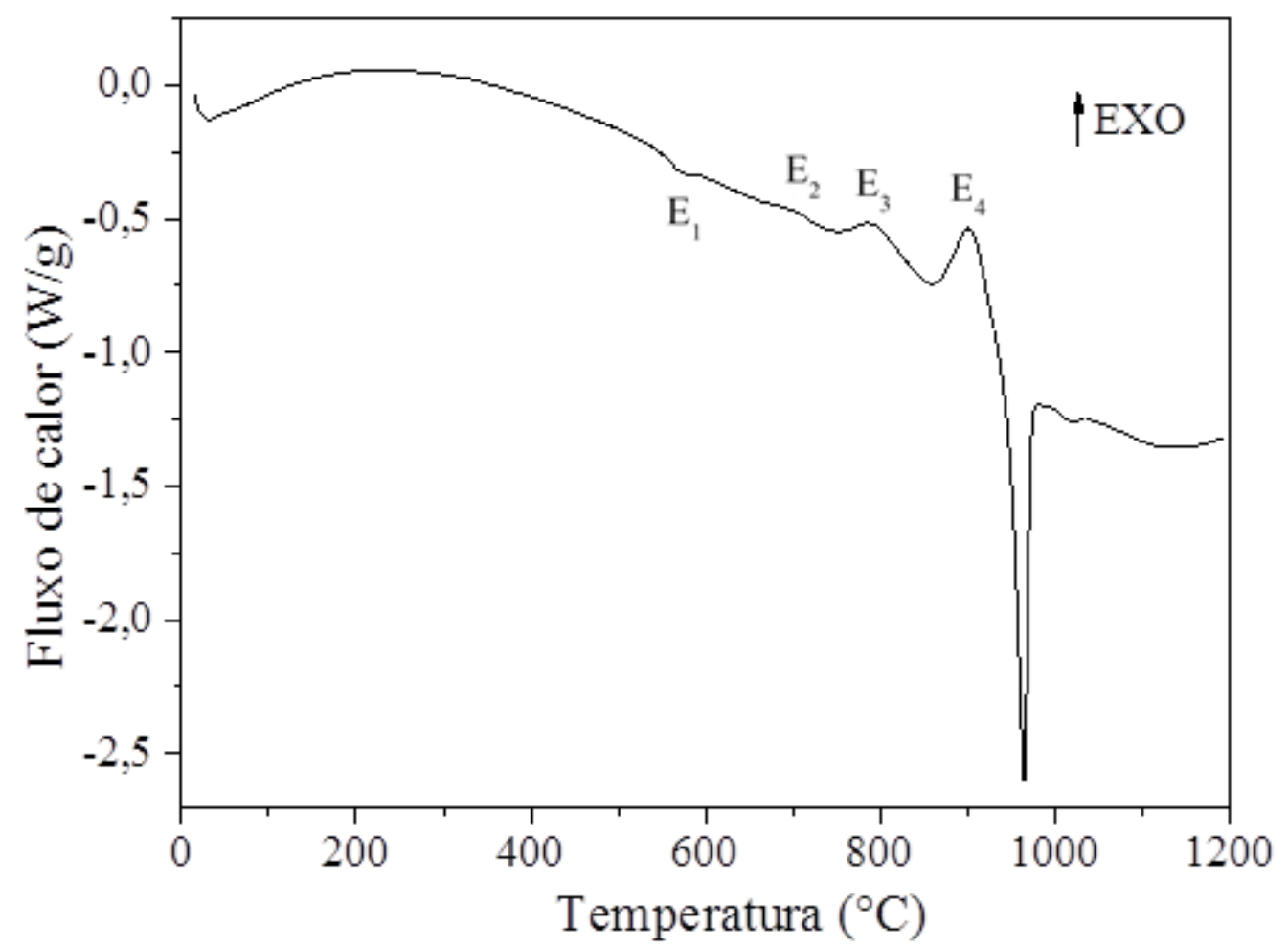

Figura 2: Calorimetria exploratória diferencial do vidro do sistema LZSA.

Pode-se observar, a partir da análise da Figura 2, a presença de uma inflexão endotérmica $\left(\mathrm{E}_{1}\right)$ a aproximadamente $590{ }^{\circ} \mathrm{C}$, característica da temperatura de transição vítrea do material. A aproximadamente 690, 790 e $850{ }^{\circ} \mathrm{C}$ pode-se observar picos exotérmicos de cristalização, identificados em trabalhos anteriores [1113], assim como as respectivas fases cristalinas formadas. Convém destacar que a formação de cristais nessa situação dá-se por meio de um processo de cristalização de superfície, no qual as partículas possuem elevada área de superfície (pó de vidro). A partir de aproximadamente $950{ }^{\circ} \mathrm{C}$, ocorre a fusão das fases cristalinas formadas e do vidro residual. A partir das informações obtidas por meio da calorimetria exploratória diferencial, Figura 2, foi possível especificar a temperatura de recozimento do vidro produzido e também estabelecer o intervalo de temperatura de tratamento térmico para cristalização superficial do vidro. A Figura 3 mostra as medidas das espessuras das camadas cristalizadas no vidro produzido em função das temperaturas e dos tempos de tratamentos térmicos utilizados.

Como podem ser observadas, pela análise da Figura 3, as espessuras das camadas cristalinas formadas aumentam aproximadamente de forma linear com a temperatura e o tempo na temperatura de tratamento térmico nos intervalos estudados. Este comportamento é compatível com outros $\mathrm{s}$

istemas vítreos estudados, conforme reportado na literatura [14].

A partir da determinação das equações das retas da Figura 3, foi possível determinar, no intervalo de temperatura e tempo estudados, a taxa ou velocidade de crescimento das camadas cristalinas formadas, conforme mostra a Tabela 2 . 


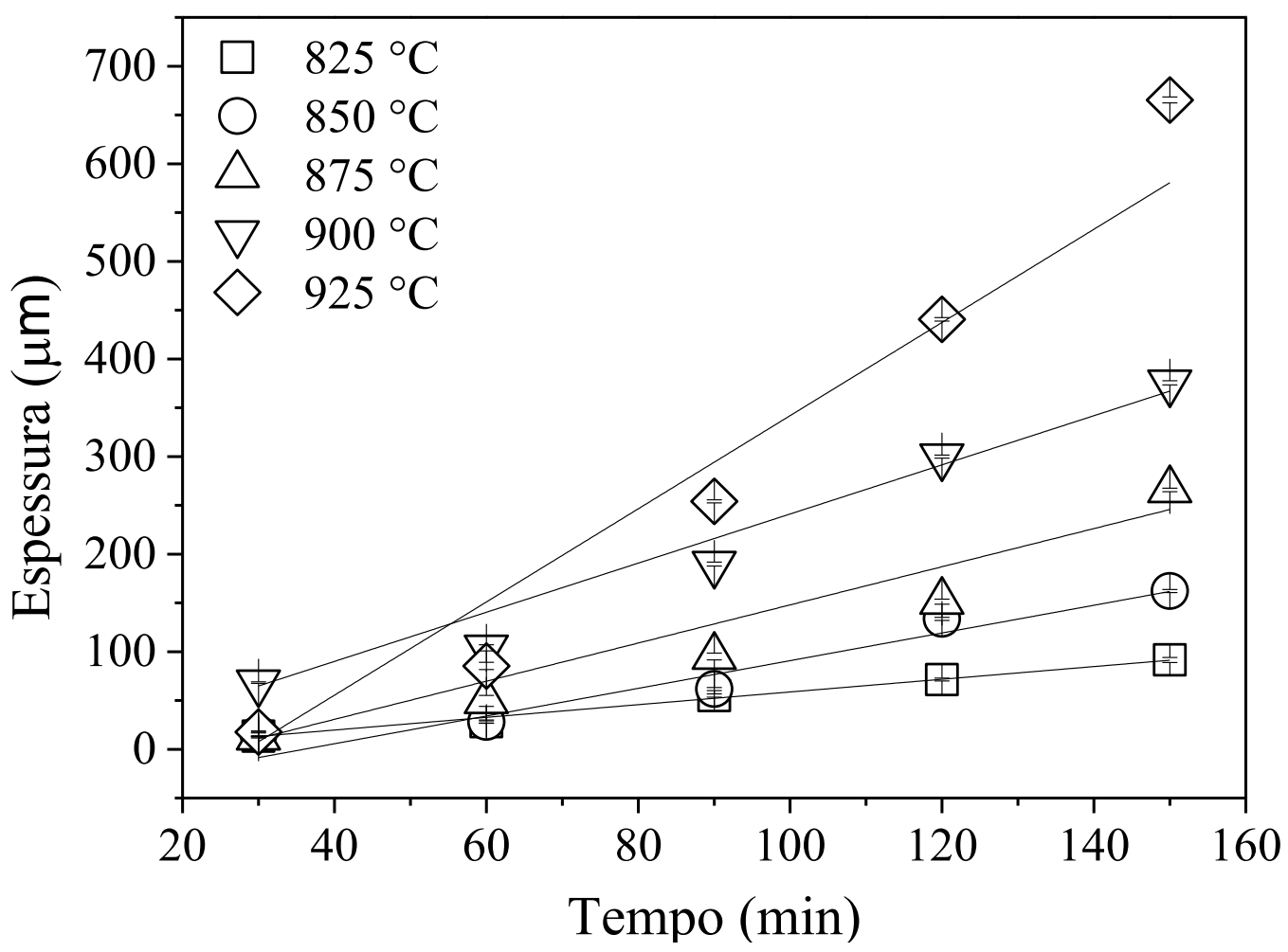

Figura 3: Variação da espessura de camada cristalizada em função da temperatura e do tempo de tratamento térmico.

Tabela 2: Evolução da taxa de crescimento das camadas cristalizadas no vidro do sistema LZSA estudado em função da temperatura.

\begin{tabular}{c|c|c}
\hline $\begin{array}{c}\text { Temperatura de tratamento } \\
\text { térmico }\left({ }^{\circ} \mathbf{C}\right)\end{array}$ & $\begin{array}{c}\text { Taxa de crescimento da camada } \\
\text { cristalizada }(\boldsymbol{\mu m} / \mathbf{m i n})\end{array}$ & $\begin{array}{c}\text { Coeficiente de } \\
\text { correlação }\left(\mathbf{R}^{\mathbf{2}}\right)\end{array}$ \\
\hline 825 & $0,69 \pm 0,05$ & 0,98 \\
850 & $1,57 \pm 0,08$ & 0,96 \\
875 & $1,96 \pm 0,06$ & 0,97 \\
900 & $2,52 \pm 0,06$ & 0,98 \\
925 & $4,80 \pm 0,07$ & 0,97 \\
\hline
\end{tabular}

Como é possível perceber pela análise da Tabela 2, as espessuras das camadas cristalinas formadas nas superfícies das amostras dos vidros aumentam rapidamente e de maneira contínua no intervalo de temperatura estudado $\left(800-925^{\circ} \mathrm{C}\right.$ ). As Figuras 4(a) e 4(b) mostram as micrografias de amostras tratadas termicamente por $120 \mathrm{~min}$ (a) e $90 \mathrm{~min}$ (b) em diferentes temperaturas, mostram camadas cristalizadas com cristais, provavelmente dendríticos ou esferulíticos, formados a partir da superfície da amostra vítrea, evidenciando desta maneira o mecanismo de cristalização superficial e a morfologia dos cristais. Tais informações permitem estabelecer ciclos térmicos para a obtenção de vidros com camadas cristalizadas com a espessura desejada.

Para as temperaturas de 825 e $850{ }^{\circ} \mathrm{C}$ durante $30 \mathrm{~min}$, conforme mostra o gráfico da Figura 3 , não foram determinadas as espessuras das camadas cristalinas formadas, já que, neste caso, o processo de crescimento das mesmas era muito incipiente e, consequentemente, as espessuras das camadas não se apresentaram uniformes ao longo da superfície analisada. Em razão disto, ao menos 10 medidas foram feitas para se determinar a espessura da camada cristalizada. De fato, em amostras tratadas a $800{ }^{\circ} \mathrm{C}$ por 30 min não foram observadas camadas cristalizadas.

A Figura 5 mostra os difratogramas de raios X (DRX) da superfície de amostras monolíticas de vidro tratadas a 825 e $900{ }^{\circ} \mathrm{C}$ por $120 \mathrm{~min}$. 


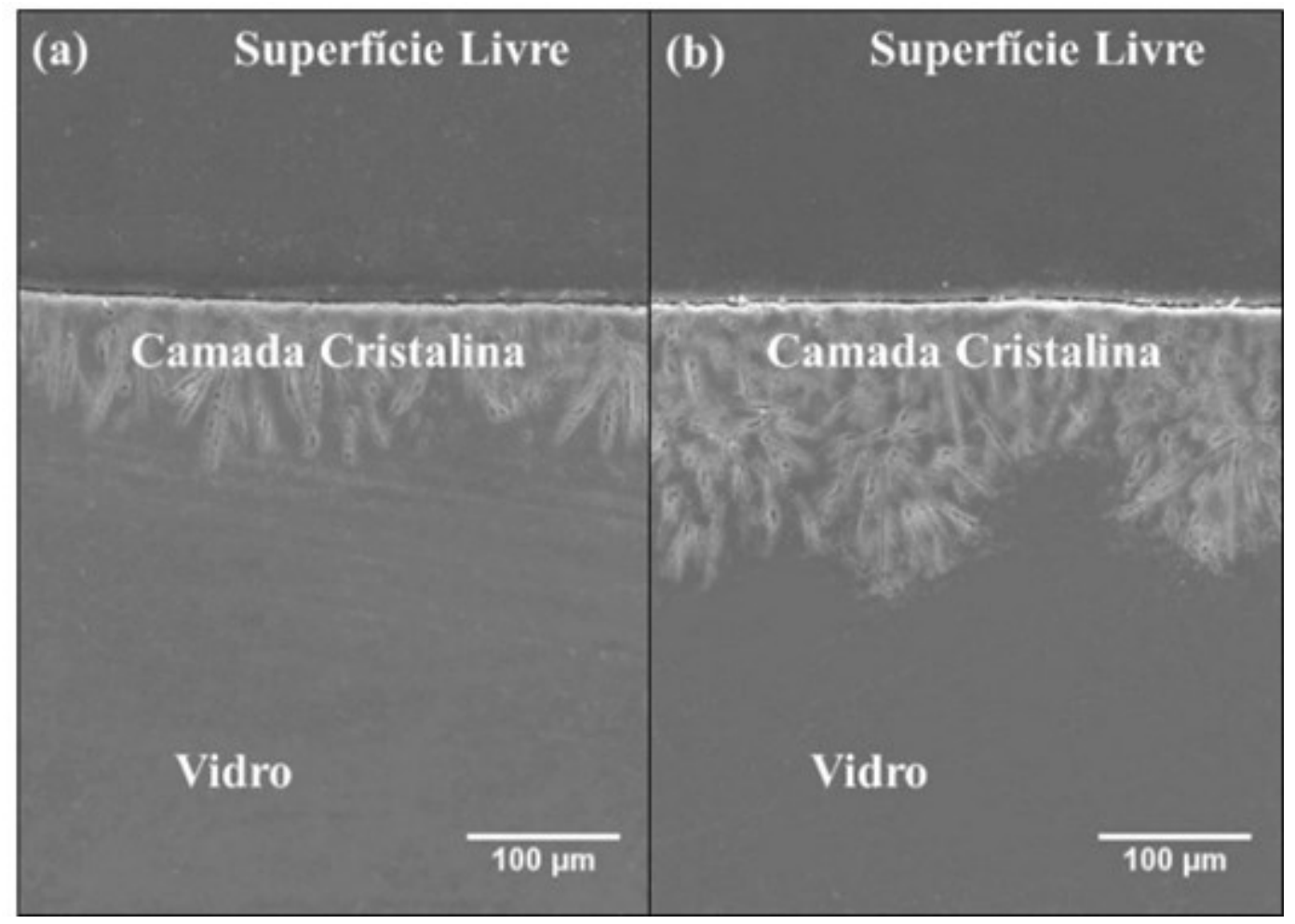

Figura 4: Micrografias (MEV) de amostras de LZSA tratadas termicamente a: (a) $825{ }^{\circ} \mathrm{C}$ por 120 min e (b) $900{ }^{\circ} \mathrm{C}$ por 90 min. Aumento de 200 X, com ataque químico (HF 2\% em volume).

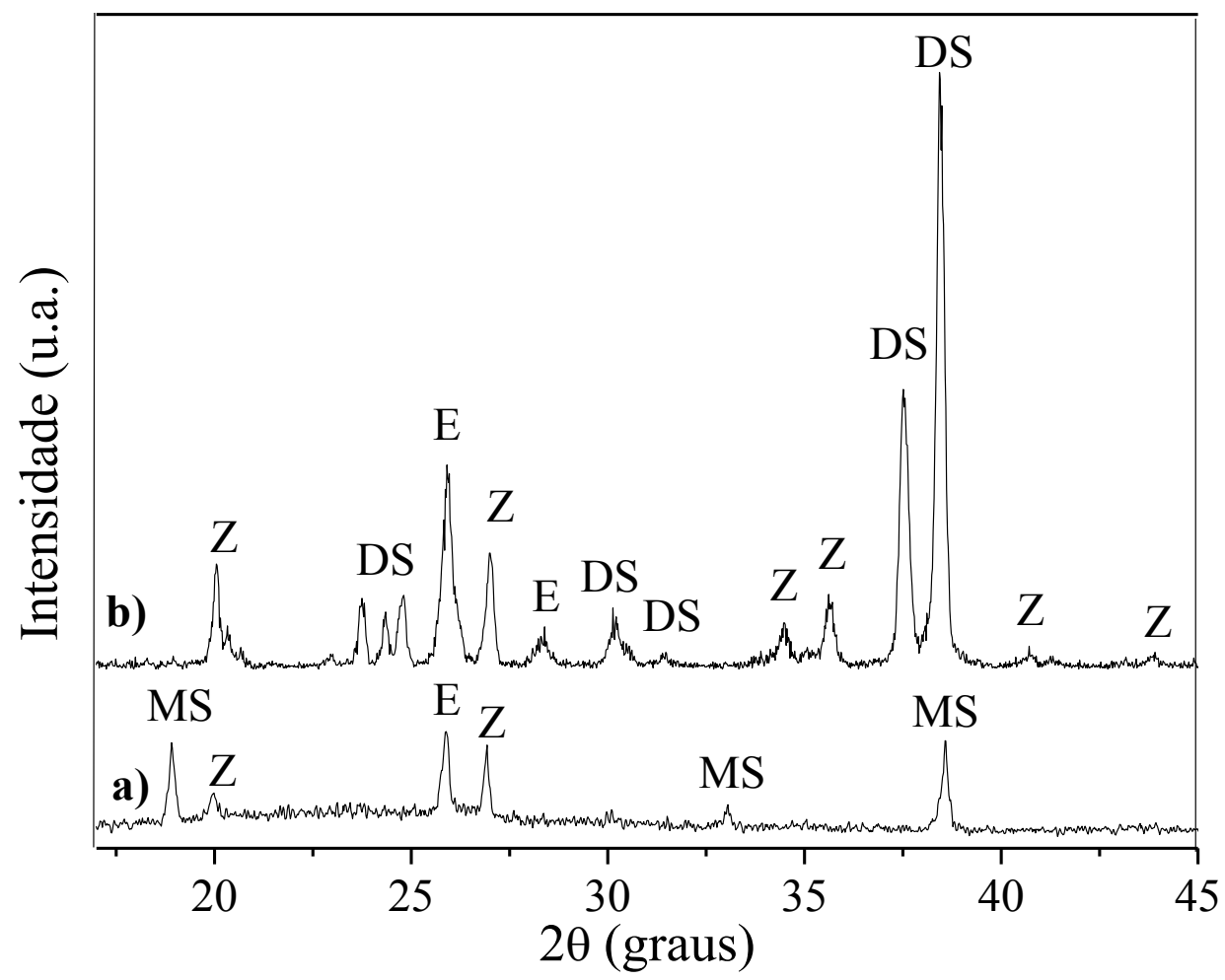

Figura 5: Difratogramas de raios $X$ das superfícies de amostras de vidros tratadas termicamente a: (a) $825^{\circ} \mathrm{C}$ por 120 min; (b) $900{ }^{\circ} \mathrm{C}$ por 120 min. $\mathrm{MS}=\mathrm{Li}_{2} \mathrm{SiO}_{3} ; \mathrm{Z}=\mathrm{ZrSiO}_{4}$; E= LiAlSi $\mathrm{O}_{6}$ (Espodumênio- $\beta$ ); $\mathrm{DS}=\mathrm{Li}_{2} \mathrm{Si}_{2} \mathrm{O}_{5}$.

A partir da análise da Figura 5 é possível perceber que o processo de cristalização superficial a $825^{\circ} \mathrm{C}$ foi apenas iniciado (picos de baixa intensidade), com a presença de espodumênio- $\beta$ ( $\mathrm{LiAlSi}_{2} \mathrm{O}_{6}$, JCPDS 35 - 


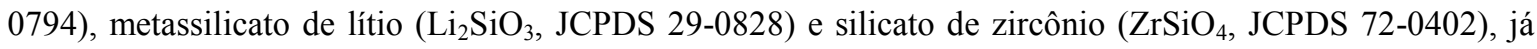
que é possível observar também uma elevação da linha de base característica do estado amorfo. Com o aumento da temperatura de tratamento térmico, isto é, $900{ }^{\circ} \mathrm{C} / 120 \mathrm{~min}$, conforme mostra a Figura 5(b), a banda amorfa não é mais evidente e o metassilicato de lítio dá lugar ao dissilicato de lítio $\left(\mathrm{Li}_{2} \mathrm{Si}_{2} \mathrm{O}_{5}\right.$, JCPDS 24-651), enquanto que a intensidade dos picos referentes às demais fases cristalinas formadas a $825{ }^{\circ} \mathrm{C}$ aumentou, indicando, provavelmente, um aumento da fração cristalizada na superfície da amostra.

\section{CONCLUSÕES}

Um vidro de composição $11,7 \mathrm{Li}_{2} \mathrm{O} .12,6 \mathrm{ZrO}_{2} \cdot 68,6 \mathrm{SiO}_{2} \cdot 7,1 \mathrm{Al}_{2} \mathrm{O}_{3}(\%$ massa $)$ foi fundido e tratado termicamente em temperaturas $\left(825-925^{\circ} \mathrm{C}\right)$ e tempos $(30-150 \mathrm{~min})$ determinados, de forma a permitir a avaliação do crescimento cristalino. Os resultados mostraram que é possível obter-se vidros do sistema LZSA com camadas cristalizadas, contendo espodumênio- $\beta$ e silicatos de zircônio e de lítio, com espessuras entre 13 e 665 $\mu \mathrm{m}$, as quais cresceram com velocidades compreendidas entre 0,4 e $4,8 \mu \mathrm{m} / \mathrm{min}$ no intervalo de temperatura de 800 a $925^{\circ} \mathrm{C}$.

\section{AGRADECIMENTOS}

Os autores agradecem os recursos fornecidos pelo PIBIC/CNPq, o projeto MCTI/CNPq/Universal 14/2014 (Processo: 444249/2014-1) e a FAPESC/CNPq (PRONEX T.O. No 17431/2011-9).

\section{BIBLIOGRAFIA}

[1] DONALD, I.W., "Review Methods for improving the mechanical properties of oxide Glasses", Journal of Materials Science, v. 24, n. 12, pp. 4177-4208, 1989.

[2] ERNSBERGER, F.M., "Strength and strengthening of glass. Part one. Strength of Glass", Glass Industry, v. 47, n. 8, pp. 422-427, 1966.

[3] GARFINKEL, H.M., "Strengthening glass by ion exchange”, Glass Industry, pp. 28-30, 1969.

[4] MACRELLI, G., "Strength issues in chemically strengthened glass", Rivista della Stazione Sperimentale del Vetro, v. 31, n. 4, pp. 69-76, 2001.

[5] ABRAMS, M.B., GREEN, D.J., GLASS, S.J. "Fracture behavior of engineered stress profile soda lime silicate glass", Journal of Non-Crystalline Solids, v. 321, n. 1-2, pp. 10-19, 2003.

[6] BRUNGS, M.P., MCCARTNEY, E.P., "Chemical strengthening of some borosilicate glasses", Physics and Chemistry of Glasses, v. 16, n. 2, pp. 44-47, 1975.

[7] MCCARTNEY, E.R., "Chemical strengthening of glass", Proceedings of the Royal Australian Chemical Institute, v. 39, pp. 175-179, 1972.

[8] VARSHNEYA, A.K., "Ion exchange: physical properties of ion-exchanged and melt-processed glasses differ”, Glass Research, v. 10, n. 11, pp. 21-26, 2001.

[9] LACOURSE, W.C., "How surface flaws affect glass strength", Glass Industry, v. 68, n. 7, pp. 14-23, 1987.

[10] BOGART, B.S., DILLIARD, P.D., US Patent 3,615,322, Anchor Hock-ing Glass Corporation Lancaster, Ohio (1971).

[11] MONTEDO, O.R.K., BERTAN, F.M., PICCOLI, R., et al., "Low thermal expansion sintered LZSA glass-ceramics", American Ceramic Society Bulletin, n. 87, pp. 34-47, 2008.

[12] MONTEDO, O.R.K., FLORIANO, F.J., FILHO, J.O., et al, "Sintering kinetics of a 18.8 $\mathrm{Li}_{2} \mathrm{O} 8.3 \mathrm{ZrO}_{2} 64.2 \mathrm{SiO}_{2} 8.7 \mathrm{Al}_{2} \mathrm{O}_{3}$ glass ceramic", Ceramics International, v. 37, pp. 1865-1871, 2011.

[13] MONTEDO, O.R.K., HOTZA, D., OLIVEIRA, A.P.N., et al, "Crystallisation Kinetics of a BSpodumene-Based Glass Ceramic", Advances in Materials Science and Engineering, v. 2012, pp. 1-8, 2012.

[14] DIAZ-MORA, N., ZANOTTO, E.D., HERGT, R., et al., "Surface Crystallization and Texture in Cordierite Glasses”, Journal of Non-Crystalline Solids, v. 273, n. 1-3, pp. 81-93, 2000. 